\title{
Distribuição ambiental de poluentes orgânicos encontrados em lodos de esgoto
}

\author{
Lourival Costa Paraíba ${ }^{(1)}$ e Maria Lúcia Saito(1)
}

(1)Embrapa Meio Ambiente, Caixa Postal 69, CEP 13820-000 Jaguariúna, SP, Brasil. E-mail: lourival@cnpma.embrapa.br, saito@cnpma.embrapa.br

Resumo - O objetivo deste trabalho foi apresentar uma modelagem da distribuição ambiental de vinte e nove poluentes orgânicos encontrados em amostras de lodos de esgoto das estações de tratamento de esgoto de Barueri e Suzano, SP. Lodos de esgoto são misturas de produtos orgânicos e inorgânicos gerados nos processos primários e secundários de tratamento que, mediante adequado controle de qualidade, podem ser reutilizados como fertilizantes agrícolas. O modelo de fugacidade nível I foi aplicado a um sistema compartimental hipotético constituído de ar, água, solo, sedimento, biota aquática e plantas. Foi utilizada a massa molecular, a pressão de vapor, a solubilidade em água, a constante de Henry, o coeficiente de partição octanol-água e a meia-vida no solo de cada um dos poluentes. O coeficiente de sorção no solo, os fatores de bioconcentração em organismos aquáticos, raízes e na seiva do xilema de plantas foram calculados por meio de expressões que correlacionam cada um desses parâmetros com o coeficiente de partição octanol-água do poluente. Foram estimados e usados os coeficientes de partição folha-ar e ar-água e o índice GUS de cada poluente. A modelagem e os cálculos dos fatores de bioconcentração e dos coeficientes de partição revelam os compartimentos preferenciais dos poluentes.

Termos para indexação: fugacidade, fertilizante, coeficiente de partição, fator de bioconcentração.

\section{Environmental distribution of the organic contaminants found in sewage sludge}

\begin{abstract}
The objective of this work was to present a model of environmental distribution of twenty-nine organic contaminants found in samples of sewage sludge of the sewage treatment plant of Barueri and Suzano SP, Brazil. Sewage sludge is a mixture of organic and inorganic products generated in primary and secondary processes of treatment that can be reused as fertilizer by the agriculture. The modeling of the contaminants distribution was accomplished by means of the level I fugacity model applied to a hypothetical compartmental system constituted by air, water, soil, sediment, aquatic biota and plants. The molecular weight, vapor pressure, water solubility, Henry's constant, octanol-water partition coefficient and soil half-life of each contaminant were used by the model. The bioconcentration factors in aquatic biota, roots, xylem sap, soil sorption coefficient were estimated by expressions that correlate each one of these bioconcentration factors with the octanol-water partition coefficient of the contaminants. The partition coefficients air-water and leaf-air, and the GUS index were calculated and used in this study. The modeling and simulations presented in this work reveal the preferential compartments of the contaminants.
\end{abstract}

Index terms: fugacity, fertilizer, partition coefficient, bioconcentration factor.

\section{Introdução}

O lodo de esgoto é um material complexo, que resulta do processo de tratamento primário e secundário de esgoto e seu descarte é motivo de preocupação no mundo todo. Além de poluentes como microrganismos e metais pesados, substâncias químicas sintéticas, provenientes de diversas fontes, podem estar presentes na solução ou podem ser sorvidas nos sólidos de lodo de esgoto. A ocorrência de poluentes orgânicos em lodo de esgoto tem crescido em virtude do aumento da produção de substâncias sintéticas, de diversas naturezas, pela indústria química. No Brasil, estudos sobre impactos ambientais dos constituintes de lodos de esgoto foram direcionados para os organismos patogênicos, metais pesados e nitratos (Andrade \& Mattiazzo, 2000; Boeira et al., 2002; Rocha et al., 2003; Vieira \& Cardoso, 2003). Segundo Tsutiya (2001), o lodo de esgoto produzido pelas estações de tratamentos de Barueri e Suzano contém poluentes orgânicos prejudiciais ao ambiente. 
Os modelos matemáticos podem contribuir para prever o destino e a preferência ambiental de poluentes e podem sugerir quais poluentes, e em quais compartimentos, devem ser sistematicamente observados em programas de monitoramento de práticas agrícolas que almejem ser economicamente e ambientalmente sustentáveis. A fugacidade, medida em unidades de pressão, descreve a tendência de escape de um composto químico orgânico em um compartimento, meio ou fase (Mackay, 1991). Os modelos de fugacidade permitem o cálculo da distribuição total da massa de um poluente entre compartimentos e são representados por um sistema linear de equações algébricas ou por um sistema linear de equações diferenciais ordinárias. A classificação nos níveis I, II, III e IV é conseqüência da complexidade dos cálculos e das hipóteses envolvidas na formulação de cada nível. Quando todas as fugacidades são iguais e constantes em todos os compartimentos, a distribuição porcentual da massa é calculada diretamente; este caso corresponde ao modelo de fugacidade nível I, ou ao modelo de fugacidade nível II, quando existem emissões, degradações e advecções do poluente em cada um dos compartimentos (Mackay, 1991). O modelo de fugacidade nível III admite que as fugacidades estão em equilíbrio estacionário e que cada compartimento pode ter diferentes valores de fugacidades, as quais são determinadas por um sistema linear de equações algébricas que descrevem as degradações, advecções, emissões e transferências (Mackay, 1991). O modelo de fugacidade nível IV descreve o comportamento não-estacionário da distribuição de um poluente entre compartimentos e permite a observação de um poluente cujas emissões e fugacidades variam com o tempo. Este tipo de modelo é descrito por um sistema de equações diferencial ordinárias ou por um sistema dinâmico de controle (Mackay, 1991; Paraíba et al., 1999).

O fator de bioconcentração (BCF) indica o grau de afinidade de um poluente a organismos vivos (Rand et al., 1995). Na estimativa do BCF para organismos aquáticos, pode ser usada a relação proposta por Isnard \& Lambert (1988). O RCF de Briggs et al. (1982) é um indicador da bioconcentração potencial de um poluente em raízes de plantas. O TSCF de Brigss et al. (1983) é um indicador da bioconcentração potencial de um poluente na seiva do xilema.

O coeficiente de sorção do poluente no carbono orgânico do solo, $\mathrm{K}_{\mathrm{oc}}$, que pode ser estimado pela relação proposta por Karickhoff (1981), mede a afinidade do poluente ao carbono orgânico do solo e pode ser usado como um indicador da afinidade do poluente pela matéria sólida do solo. O coeficiente de partição folha-ar, $\mathrm{K}_{\mathrm{la}}$, é um indicador da afinidade de um poluente por folhas. O coeficiente de partição ar-água, $K_{a w}$, pode ser usado para identificar os poluentes voláteis. Todos estes indicadores estão correlacionados com o coeficiente de partição octanol-água, $\mathrm{K}_{\text {ow }}$, do poluente, o qual exprime a afinidade de um poluente por lipídio. O potencial de lixiviação de um poluente orgânico é freqüentemente estimado com o auxílio do índice GUS de Gustafson (1989), o qual compõe, em seus cálculos, o coeficiente de sorção e a meia-vida do poluente no solo.

Os objetivos deste trabalho foram modelar a distribuição ambiental de vinte e nove poluentes orgânicos entre os compartimentos de um sistema ambiental hipotético, e aplicar indicadores do comportamento ambiental de compostos químicos orgânicos, para identificar os compartimentos preferenciais de vinte e nove poluentes orgânicos encontrados em amostras de lodos de esgotos das estações de tratamentos localizadas nos municípios de Barueri e Suzano, SP.

\section{Material e Métodos}

O modelo de fugacidade nível I, descrito a seguir, foi utilizado para simular a distribuição porcentual de um conjunto de poluentes entre os compartimentos de um sistema ambiental hipotético constituído de ar, água, solo, sedimento, biota aquática e plantas. O modelo de fugacidade nível I é recomendado pela Organization for Economic Cooperation and Development (OECD, 1999) aos países membros como uma das estratégias de análise da exposição ambiental às substâncias químicas.

A fugacidade de um poluente está relacionada com a concentração $\mathrm{C}\left(\mathrm{mol} \mathrm{m}^{-3}\right)$ por meio da capacidade de fugacidade $\mathrm{Z}$ ( $\mathrm{mol} \mathrm{m}^{-3} \mathrm{~Pa}^{-1}$ ) do compartimento para o poluente. A concentração é dada pela relação $\mathrm{C}=\mathrm{Zf}$, em que f ( $\mathrm{Pa}$ ) é a fugacidade do poluente no compartimento. Em estado de equilíbrio de fugacidades, os compartimentos com altas capacidades de fugacidade terão altas concentrações do poluente. Quando as fugacidades são iguais e constantes $\left(f_{i}=f_{j}=f\right)$, pode-se calcular o coeficiente de partição do poluente entre os compartimentos i e $\mathrm{j}, \mathrm{K}_{\mathrm{ij}}$, pelo quociente $\mathrm{K}_{\mathrm{ij}}=\mathrm{Z}_{\mathrm{i}} / \mathrm{Z}_{\mathrm{j}}$ em que $\mathrm{Z}_{\mathrm{i}}$ é a capacidade de fugacidade do compartimento i, e $Z_{j}$ é a capacidade de fugacidade do compartimento $\mathrm{j}$ (Mackay, 1991).

A capacidade de fugacidade do ar é definida pela expressão: 
$Z_{\mathrm{a}}=\frac{1}{\mathrm{R}(273+\mathrm{t})}+\frac{0,12 \times 10^{-3}}{\mathrm{R}(273+\mathrm{t}) \mathrm{p}_{\mathrm{v}}}$ em que $\mathrm{R}$ é a constante dos gases $\left(8,314 \mathrm{~Pa} \mathrm{~m}^{3} \mathrm{~mol}^{-1} \mathrm{~K}^{-1}\right)$, t $\left({ }^{\circ} \mathrm{C}\right)$ é a temperatura ambiente e $\mathrm{p}_{\mathrm{v}}(\mathrm{Pa})$ é a pressão de vapor do poluente (Mackay et al., 1996).

A capacidade de fugacidade da água é calculada pela expressão:

$\mathrm{Z}_{\mathrm{W}}=\frac{1}{\mathrm{H}}+\frac{5,0 \times 10^{-3} \rho_{\mathrm{sd}} \mathrm{v}_{\mathrm{oc}}^{\mathrm{sd}} \mathrm{K}_{\mathrm{oc}}}{\mathrm{H}}+\frac{10^{-3} \rho_{\mathrm{b}} \mathrm{v}_{\mathrm{b}} \mathrm{K}_{\mathrm{ow}}}{\mathrm{H}}$

em que $\mathrm{H}\left(\mathrm{Pa} \mathrm{m}^{3} \mathrm{~mol}^{-1}\right)$ é a constante de Henry, $\mathrm{r}_{\mathrm{sd}}$ $\left(\mathrm{kg} \mathrm{m}^{-3}\right)$ é a densidade das partículas de sedimento em suspensão na água, $\mathrm{v}_{\mathrm{oc}}^{\text {sd }}$ é a fração volumétrica de carbono orgânico no sedimento em suspensão, $K_{o c}$ $\left(\mathrm{m}^{3} \mathrm{~kg}^{-1}\right)$ é o coeficiente de sorção no carbono orgânico do sedimento, $\rho_{\mathrm{b}}\left(\mathrm{kg} \mathrm{m}^{-3}\right)$ é a densidade da biota aquática, $\mathrm{v}_{\mathrm{b}}$ é a fração volumétrica da biota aquática e $\mathrm{K}_{\mathrm{ow}}$ é o coeficiente de partição octanol-água (Mackay et al., 1996).

A capacidade de fugacidade do solo foi estimada pela expressão:

$\mathrm{Z}_{\mathrm{sl}}=\frac{0,2}{\mathrm{R}(273+\mathrm{t})}+\frac{0,3}{\mathrm{H}}+\frac{0,5 \mathrm{v}_{\mathrm{oc}}^{\mathrm{sl}} \rho_{\mathrm{sl}} \mathrm{K}_{\mathrm{oc}}}{\mathrm{H}}$

em que $\mathrm{v}_{\mathrm{oc}}^{\mathrm{sl}}$ é o conteúdo volumétrico de carbono orgânico do solo e $\rho_{\mathrm{sl}}\left(\mathrm{kg} \mathrm{m}^{-3}\right)$ é a densidade do solo (Mackay et al., 1996). Os valores 0,2, 0,3 e 0,5 na expressão de $\mathrm{Z}_{\mathrm{sl}}$ correspondem, respectivamente, ao conteúdo volumétrico de ar, água e matéria sólida de um solo hipotético.

A capacidade do sedimento é estimada pela expressão:

$\mathrm{Z}_{\mathrm{sd}}=\frac{0,8}{\mathrm{H}}+\frac{0,2 \rho_{\mathrm{sd}} \mathrm{v}_{\mathrm{oc}}^{\mathrm{sd}} \mathrm{K}_{\mathrm{oc}}}{\mathrm{H}}$

(Mackay et al., 1996). Os valores 0,8 e 0,2 na expressão de $\mathrm{Z}_{\text {sd }}$ correspondem, respectivamente, ao conteúdo volumétrico de água e de matéria sólida do sedimento.

A capacidade de fugacidade da biota aquática é estimada pela expressão:

$Z_{b}=\frac{10^{-3} \rho_{b} v_{b} K_{o w}}{H}$ (Mackay et al., 1996).

A capacidade de fugacidade do compartimento planta foi estimada admitindo-se que as plantas são constituídas por $10 \%$ de folhas, $40 \%$ de raízes e $50 \%$ de caule e que é dada pela expressão $Z_{p}=0,1 Z_{1}+0,4 Z_{r}+0,5 Z_{c}$.
Estas capacidades de fugacidade são dadas pelas expressões:

$\mathrm{Z}_{\mathrm{l}}=\frac{0,2}{\mathrm{R}(273+\mathrm{t})}+\left(\frac{0,78}{\mathrm{H}}+\frac{0,02 \mathrm{~K}_{\mathrm{ow}}}{\mathrm{H}}\right) \frac{\rho_{\mathrm{l}}}{\rho_{\mathrm{w}}}$

$\mathrm{Z}_{\mathrm{r}}=\left(\frac{0,82}{\mathrm{H}}+0,02 \mathrm{~K}_{\mathrm{ow}} \mathrm{Z}_{\mathrm{s}}\right) \frac{\rho_{\mathrm{r}}}{\rho_{\mathrm{w}}}$

$Z_{c}=\left(\frac{0,82}{H}+0,014 K_{o w} Z_{w}\right) \frac{\rho_{c}}{\rho_{w}}$

em que $Z_{1}$ é a capacidade de fugacidade da folha, $Z_{r}$ é a capacidade de fugacidade da raiz, $Z_{c}$ é a capacidade de fugacidade do caule e $\rho_{\mathrm{l}}, \rho_{\mathrm{r}}$ e $\rho_{\mathrm{c}}\left(\mathrm{kg} \mathrm{m}^{-3}\right)$ são a densidade da folha, da raiz e do caule, respectivamente (Trapp \& McFarlane, 1995; Cousins \& Mackay, 2001). Os valores 0,78, 0,82 e 0,82 correspondem, respectivamente, ao conteúdo volumétrico de água na folha, na raiz e no caule de uma planta hipotética. Os valores 0,02, 0,02 e 0,014 correspondem, respectivamente, ao conteúdo volumétrico de lipídio de folha, raiz e caule de uma planta hipotética. O valor 0,2 na expressão de $Z_{1}$ corresponde ao conteúdo volumétrico de ar na folha de uma planta hipotética.

No modelo de fugacidade nível I, supõe-se que as fugacidades são iguais e constantes em todos os compartimentos, ou seja, $\left(f_{i}=f_{j}=f\right)$ para todo $i, j \in J$, $\mathrm{J}=\{\mathrm{a}, \mathrm{w}, \mathrm{sl}, \mathrm{sd}, \mathrm{b}, \mathrm{p}\}$, as capacidades de fugacidade são determinadas e os processos de transferências dinâmicas do poluente entre compartimentos e as taxas de transformações do poluente em cada compartimento são hipoteticamente nulas. Nessas condições, se cada compartimento $\mathrm{i} \in \mathrm{J}$ tiver um volume bem definido $\mathrm{V}_{\mathrm{i}}$, então o porcentual do poluente j no compartimento i é estimado pela expressão:

$P_{i}^{j}=\frac{Z_{i}^{j} V_{i}}{\sum_{i \in J} Z_{i}^{j} V_{i}} \times 100$

em que $l \leq j \leq 29$ são os índices representando os poluentes e $Z_{i}^{j}$ é a capacidade de fugacidade do compartimento i para o poluente $j$. Dessa forma, os maiores valores $P_{i}^{j}$ revelam os compartimentos preferenciais do poluente $\mathrm{j}$. Assim, admitindo-se a hipótese de que as fugacidades do poluente no sistema hipotético são iguais e constantes, os cálculos do modelo de fugacidade nível I esti- 
mam a distribuição volumétrica do poluente entre os compartimentos do sistema modelado.

O potencial de lixiviação de cada poluente foi estimado mediante o cálculo do índice:

GUS $=\left[4-\log \left(10^{3} \mathrm{~K}_{\mathrm{oc}}\right)\right] \times \log \left(\mathrm{t}_{1 / 2}^{\mathrm{sl}}\right)$

em que $K_{o c}\left(m^{3} \mathrm{~kg}^{-1}\right)$ é o coeficiente de sorção e $t_{1 / 2}^{s l}$ (dias) é a meia-vida do poluente no solo. $O$ valor $10^{3}$ na expressão do índice GUS é conseqüência da unidade adotada neste trabalho para o valor de $\mathrm{K}_{\mathrm{oc}}$, $\mathrm{em} \mathrm{m}^{3} \mathrm{~kg}^{-1}$.

O coeficiente de sorção, em $\mathrm{m}^{3} \mathrm{~kg}^{-1}$, foi estimado pela relação:

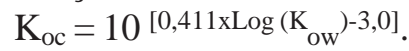

Conforme o valor numérico do índice GUS, o poluente é um potencial lixiviante (GUS $\geq 2,8$ ), um potencial nãolixiviante (GUS $\leq 1,8$ ) ou um poluente de lixiviação indeterminada $(1,8 \leq \mathrm{GUS} \leq 2,8)$.

O fator de bioconcentração em organismos aquáticos ou na biota aquática (BCF) foi estimado pela rela-

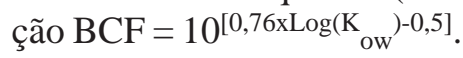

O fator de bioconcentração na raiz (RCF) foi estima-

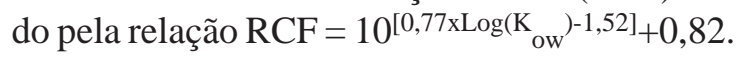

A bioconcentração na seiva do xilema (TSCF) foi estimada pela relação:

$\mathrm{TSCF}=0,784 \times \exp \left[\frac{-\left(\log \left(\mathrm{K}_{\mathrm{ow}}\right)-1,78\right)^{2}}{2,44}\right]$.

A expressão para o TSCF indica que para os poluentes com valores de $\mathrm{K}_{\text {ow }}$ ao redor de 60 ou $\log \left(\mathrm{K}_{\text {ow }}\right) \cong 1,78$, o potencial de bioconcentração do poluente na seiva do xilema de plantas é máximo (TSCF $=0,748)$ (Trapp \& Matthies, 1998), ou seja, a absorção do poluente pela raiz no processo de transpiração é máximo.

Um indicador da afinidade do poluente por folhas de plantas é dado pelo coeficiente de partição folha-ar, descrito pelo quociente:

$\mathrm{K}_{\mathrm{la}}=\frac{\mathrm{Z}_{1}}{\mathrm{Z}_{\mathrm{a}}}$

em que $Z_{1}$ é a capacidade de fugacidade da folha e $Z_{a}$ é a capacidade de fugacidade do ar. Este indicador é geralmente usado para selecionar poluentes contaminantes de folhas por deposição de partículas contaminadas da atmosfera (Trapp \& Matthies, 1995).

O coeficiente de partição ar-água é um indicador da volatilização de um poluente e é dado por:

$\mathrm{K}_{\mathrm{aw}}=\frac{\mathrm{H}}{\mathrm{R}(273+\mathrm{t})}$ em que H é a constante de Henry, R é a constante dos gases e t é a temperatura ambiente.

O volume dos compartimentos ar, água, solo, sedimento, biota aquática e planta do sistema ambiental adotado neste trabalho foi de $10^{8} \mathrm{~m}^{3}, 2,0.10^{5} \mathrm{~m}^{3}, 9,0.10^{5} \mathrm{~m}^{3}$, $10^{4} \mathrm{~m}^{3}, 1,0 \mathrm{~m}^{3}$ e $9,0.10^{2} \mathrm{~m}^{3}$, respectivamente. O volume de ar corresponde a um meio aéreo de $10^{6} \mathrm{~m}^{2}$ de área por $10^{2} \mathrm{~m}$ de altura. $\mathrm{O}$ volume de água corresponde a um meio aquático de $10^{5} \mathrm{~m}^{2}$ de área por $2,0 \mathrm{~m}$ de profundidade. $\mathrm{O}$ volume de solo corresponde a um meio edáfico com $9,0.10^{5} \mathrm{~m}^{2}$ de área por $1,0 \mathrm{~m}$ de profundidade. $\mathrm{O}$ volume de sedimento corresponde ao fundo de um ambiente aquático com uma área de $10^{5} \mathrm{~m}^{2}$ por $0,1 \mathrm{~m}$ de profundidade. As densidades de água, solo, sedimento, folha, caule, raiz e biota aquáticas foram estimadas em $989 \mathrm{~kg} \mathrm{~m}^{-3}, 1.500 \mathrm{~kg} \mathrm{~m}^{-3}, 1.400 \mathrm{~kg} \mathrm{~m}^{-3}, 820 \mathrm{~kg} \mathrm{~m}^{-3}$, $850 \mathrm{~kg} \mathrm{~m}^{-3}, 820 \mathrm{~kg} \mathrm{~m}^{-3}$ e $1.100 \mathrm{~kg} \mathrm{~m}^{-3}$, respectivamente. Foram admitidos os valores de 0,018, 0,02 e 0,056 para as frações volumétricas de carbono orgânico do solo, do sedimento e da biota aquática, respectivamente.

Foram selecionados vinte e nove poluentes orgânicos encontrados em amostras dos lodos de esgotos de Barueri e Suzano (Tsutiya, 2001). Em Syracuse Research Corporation (SRC, 2005) foram coletados o peso molecular, a pressão de vapor, a constante de Henry, a solubilidade em água e o coeficiente de partição octanol-água. A meia-vida do poluente no solo foi retirada de Howard et al. (1991). A Tabela 1 apresenta a compilação dos dados físico-químicos dos poluentes utilizados neste trabalho.

Os cálculos foram realizados usando-se uma planilha Excel de MS-Office98 contendo as características físico-químicas de cada poluente e as expressões numéricas que calcularam as capacidades de fugacidade, a distribuição porcentual, os fatores de bioconcentração, os coeficientes de partição e os índices GUS.

\section{Resultados e Discussão}

Alguns poluentes são pouco solúveis em água (Tabela 1). Em geral, a solubilidade e a pressão de vapor diminuem com o aumento da massa molecular. Muitos poluentes apresentam valores de $\mathrm{K}_{\mathrm{ow}}$ superiores a 1.000, 
demonstrando grande afinidade lipofílica do conjunto de poluentes. Estas propriedades indicam que alguns desses poluentes podem ser encontrados tanto na fase gasosa do ar quanto sorvidos em partículas orgânicas sólidas ou em partículas contendo carbono orgânico em suspensão no ar.

A meia-vida no solo dos poluentes de maior massa molecular é relativamente maior e, em virtude do valor do coeficiente de sorção, alguns desses poluentes no ambiente seriam possivelmente encontrados sorvidos na matriz sólida do solo. Aqueles poluentes cuja meia-vida no solo é relativamente alta e o coeficiente de sorção no solo é relativamente baixo, ou com alta solubilidade em água, são classificados como poluentes lixiviantes por causa do valor do índice GUS $\geq 2,8$ (Tabelas 2 e 3). Existe uma correlação positiva entre o valor do coeficiente de sorção $\mathrm{K}_{\mathrm{oc}}$ e o porcentual de distribuição do poluente no solo e ou no sedimento, e uma correlação positiva entre o potencial de lixiviação com o porcentual de distribui- ção na água (Tabelas 2 e 3). Em virtude da capacidade de fugacidade, o porcentual de distribuição no solo exprime a afinidade do poluente na fração gasosa do solo, na matriz sólida e na solução aquosa.

Em geral, poluentes com $\mathrm{K}_{\mathrm{aw}} \leq 4,0.10^{-6}$ não são voláteis e apresentam baixos porcentuais de distribuição no ar. Poluentes com $4,0.10^{-6}<\mathrm{K}_{\mathrm{aw}}<4,0.10^{-4}$ têm a volatilização controlada por difusão gasosa e dependente da temperatura e da umidade, e apresentam baixos porcentuais de distribuição no ar. Os poluentes com $\mathrm{K}_{\mathrm{aw}} \geq 4,0.10^{-4}$ são provavelmente voláteis e podem apresentar altos porcentuais de distribuição no ar (Trapp \& Matthies, 1998). É importante observar que alguns dos poluentes classificados como potencialmente voláteis apresentam baixa afinidade por folha (Tabelas 2 e 3). Segundo Cousins \& Mackay (2001), poluentes com $\log \left(k_{0 a}\right) \geq 6$ e $\log \left(k_{a w}\right) \leq-6$ são possivelmente absorvidos do ar pelas plantas, em que $\mathrm{K}_{\mathrm{oa}}=\frac{\mathrm{K}_{\mathrm{ow}}}{\mathrm{K}_{\mathrm{aw}}}$ é o coeficiente de partição octanol-ar do poluente (Bacci, 1994).

Tabela 1. Propriedades físico-químicas dos vinte e nove poluentes orgânicos encontrados em amostras dos lodos de esgoto de Barueri e Suzano, SP.

\begin{tabular}{|c|c|c|c|c|c|c|}
\hline Poluente & $\begin{array}{c}\text { Massa molecular } \\
\left(\mathrm{g} \mathrm{mol}^{-1}\right)\end{array}$ & $\begin{array}{l}\text { Solubilidade em água } \\
\left(\mathrm{g} \mathrm{m}^{-3}\right)\end{array}$ & $\begin{array}{c}\log \\
\left(\mathrm{K}_{\mathrm{ow}}\right)\end{array}$ & $\begin{array}{l}\text { Pressão de vapor } \\
(\mathrm{Pa})\end{array}$ & $\begin{array}{c}\text { Constante de Henry } \\
\left(\mathrm{Pa} \mathrm{m}^{-3} \mathrm{~mol}^{-1}\right)\end{array}$ & $\begin{array}{c}\text { Meia-vida no } \\
\text { solo (dias) }\end{array}$ \\
\hline 1,2,4-triclorobenzeno & 181,45 & $4,90 \mathrm{E}+01$ & 4,02 & $6,13 \mathrm{E}+01$ & $1,44 \mathrm{E}+02$ & 28 \\
\hline 1,2-diclorobenzeno & 147,00 & $1,56 \mathrm{E}+02$ & 3,43 & $1,81 \mathrm{E}+02$ & $1,95 \mathrm{E}+02$ & 180 \\
\hline 3-diclorobenzeno & 147,00 & $1,25 \mathrm{E}+02$ & 3,53 & $2,87 \mathrm{E}+02$ & $2,66 \mathrm{E}+02$ & 180 \\
\hline 1,4-diclorobenzeno & 147,00 & $8,13 \mathrm{E}+01$ & 3,44 & $2,32 \mathrm{E}+02$ & $2,44 \mathrm{E}+02$ & 365 \\
\hline 2,4-dinitrofenol & 184,11 & $2,79 \mathrm{E}+03$ & 1,67 & $5,20 \mathrm{E}-02$ & $8,71 \mathrm{E}-03$ & 263 \\
\hline 2-clorofenol & 128,56 & $1,13 \mathrm{E}+04$ & 2,15 & $3,37 \mathrm{E}+02$ & $1,13 \mathrm{E}+00$ & 3 \\
\hline 3,3'-diclorobenzidina & 253,13 & $3,10 \mathrm{E}+00$ & 3,51 & $3,41 \mathrm{E}-05$ & $2,88 \mathrm{E}-06$ & 180 \\
\hline Antraceno & 178,24 & $4,34 \mathrm{E}-02$ & 4,45 & $3,56 \mathrm{E}-04$ & $5,63 \mathrm{E}+00$ & 459 \\
\hline Benzo(a)antraceno & 228,30 & $9,40 \mathrm{E}-03$ & 5,76 & $2,53 \mathrm{E}-04$ & $1,22 \mathrm{E}+00$ & 579 \\
\hline Benzidina & 184,24 & $3,22 \mathrm{E}+02$ & 1,34 & $1,20 \mathrm{E}-04$ & $5,24 \mathrm{E}-06$ & 8 \\
\hline Benzo(a)pireno & 252,32 & $1,62 \mathrm{E}-03$ & 6,13 & $7,32 \mathrm{E}-07$ & $4,63 \mathrm{E}-02$ & 529 \\
\hline Benzo(b)fluoranteno & 252,32 & $1,50 \mathrm{E}-03$ & 5,78 & $6,67 \mathrm{E}-05$ & $6,66 \mathrm{E}-02$ & 659 \\
\hline Benzo(k)fluoranteno & 252,32 & $8,00 \mathrm{E}-04$ & 6,11 & $1,29 \mathrm{E}-07$ & $5,92 \mathrm{E}-02$ & 2.108 \\
\hline $\operatorname{Dibenzo}(\mathrm{a}, \mathrm{h})$ antraceno & 278,36 & $2,49 \mathrm{E}-03$ & 6,75 & $1,33 \mathrm{E}-08$ & $1,25 \mathrm{E}-02$ & 1.001 \\
\hline Difenilnitrosamina & 198,23 & $3,50 \mathrm{E}+01$ & 3,13 & $1,33 \mathrm{E}+01$ & $1,23 \mathrm{E}-01$ & 68 \\
\hline Hexaclorobenzeno & 284,78 & $6,20 \mathrm{E}-03$ & 5,73 & $2,40 \mathrm{E}-03$ & $1,72 \mathrm{E}+02$ & 2.380 \\
\hline Hexaclorobutadieno & 260,76 & $3,20 \mathrm{E}+00$ & 4,78 & $2,93 \mathrm{E}+01$ & $1,04 \mathrm{E}+03$ & 180 \\
\hline Hexaclorociclopentadieno & 272,77 & $1,80 \mathrm{E}+00$ & 5,04 & $8,00 \mathrm{E}+00$ & $2,74 \mathrm{E}+03$ & 28 \\
\hline Hexacloroetano & 236,74 & $5,00 \mathrm{E}+01$ & 4,14 & $2,80 \mathrm{E}+01$ & $3,94 \mathrm{E}+02$ & 200 \\
\hline Hidrazobenzeno & 184,24 & $2,21 \mathrm{E}+02$ & 2,94 & $5,81 \mathrm{E}-02$ & $4,45 \mathrm{E}-04$ & 180 \\
\hline Indeno(1,2,3-cd)pireno & 276,34 & $1,90 \mathrm{E}-04$ & 6,70 & $1,67 \mathrm{E}-08$ & $3,53 \mathrm{E}-02$ & 790 \\
\hline Isoforona & 138,21 & $1,20 \mathrm{E}+04$ & 1,70 & $5,84 \mathrm{E}+01$ & $6,73 \mathrm{E}-01$ & 28 \\
\hline Naftaleno & 128,18 & $3,10 \mathrm{E}+01$ & 3,30 & $1,13 \mathrm{E}+01$ & $4,46 \mathrm{E}+01$ & 48 \\
\hline Nitrobenzeno & 123,11 & $2,09 \mathrm{E}+03$ & 1,85 & $3,27 \mathrm{E}+01$ & $2,43 \mathrm{E}+00$ & 198 \\
\hline n-nitrosodipropilamina & 130,19 & $1,30 \mathrm{E}+04$ & 1,36 & $1,15 \mathrm{E}+01$ & $5,45 \mathrm{E}-01$ & 180 \\
\hline Pentaclorofenol & 266,34 & $1,40 \mathrm{E}+01$ & 5,12 & $1,47 \mathrm{E}-02$ & $2,48 \mathrm{E}-03$ & 178 \\
\hline Fenantreno & 178,24 & $1,15 \mathrm{E}+00$ & 4,46 & $1,49 \mathrm{E}-02$ & $4,29 \mathrm{E}+00$ & 200 \\
\hline Fenol & 94,11 & $8,28 \mathrm{E}+04$ & 1,46 & $4,67 \mathrm{E}+01$ & $3,37 \mathrm{E}-02$ & 10 \\
\hline Pireno & 202,26 & $1,35 \mathrm{E}-01$ & 4,88 & $6,00 \mathrm{E}-04$ & $1,21 \mathrm{E}+00$ & 2.133 \\
\hline
\end{tabular}


Segundo a OECD (1981), poluentes orgânicos prejudiciais à cadeia alimentar via bioconcentração na biota aquática apresentam valores de $\mathrm{K}_{\mathrm{ow}}$ entre $10^{2}$ e $10^{6}$ $\left(2,0 \leq \log \left(K_{\text {ow }}\right) \leq 6,0\right)$ e solubilidade em água menor do que $2 \mathrm{~g} \mathrm{~m}^{-3}$ (Tabela 3). Este mesmo critério foi adotado para selecionar os poluentes que apresentam potencial de bioconcentração em raiz, dado pelo valor do RCF (Tabela 3). Os valores numéricos de RCF e TSCF indicam que se um poluente apresenta risco de bioconcentração em raiz, então não apresenta risco de bioconcentração na seiva do xilema e, reciprocamente, se um poluente apresenta risco de bioconcentração na seiva do xilema, então o poluente não apresenta risco de bioconcentração em raiz. Porém, em qualquer um dos casos, para que um poluente seja considerado crítico para plantas é necessário que o mesmo esteja no solo biologicamente disponível para plantas (Tabela 3).

Segundo Trapp \& Matthies (1995), compostos orgânicos $\operatorname{com} \log \left(\mathrm{K}_{\mathrm{la}}\right) \geq 7$ são poluentes prejudiciais às plantas, pois, contaminam folhas por deposição de partícu- las da atmosfera sorvidas com o poluente (Tabela 3). Como o coeficiente de partição folha-ar está positivamente relacionado com o coeficiente de partição octanolágua, mediante a capacidade de fugacidade da folha, e inversamente proporcional ao coeficiente de partição arágua, por meio da capacidade de fugacidade do ar, alguns poluentes podem ter altos valores de $\mathrm{K}_{\mathrm{la}}$ tendo altos valores de RCF, sugerindo que os poluentes que têm afinidade por raiz também podem apresentar alta afinidade por folhas (Tabelas 2 e 3).

Na escolha de poluentes como indicadores para monitorar a qualidade de um determinado lodo de esgoto para fins agrícolas, deveriam ser escolhidos os seguintes: benzo(a)antraceno, benzo(a)pireno, benzo(b)fluoranteno, benzo(k)fluoranteno e dibezo(a,h)antraceno, pois, estes poluentes orgânicos são carcinogênicos, genotóxicos ou mutagênicos para o ser humano (Pereira Netto et al., 2000), e possuem altos porcentuais de distribuição em plantas (Tabela 2).

Tabela 2. Distribuição ambiental porcentual dos vinte e nove poluentes orgânicos encontrados em amostras dos lodos de esgotos de Barueri e Suzano, SP, calculada mediante o modelo de fugacidade nível I.

\begin{tabular}{|c|c|c|c|c|c|c|}
\hline Poluente & $\begin{array}{c}\mathrm{Ar} \\
(\%)\end{array}$ & $\begin{array}{l}\text { Água } \\
(\%)\end{array}$ & $\begin{array}{l}\text { Solo } \\
(\%)\end{array}$ & $\begin{array}{c}\text { Biota aquática } \\
(\%)\end{array}$ & $\begin{array}{c}\text { Sedimento } \\
(\%)\end{array}$ & $\begin{array}{c}\text { Planta } \\
(\%)\end{array}$ \\
\hline 1,2,4-triclorobenzeno & 8,90 & 0,49 & 83,91 & $9,36 \mathrm{E}-04$ & 0,4245 & 6,27 \\
\hline 1,2-diclorobenzeno & 35,49 & 1,04 & 61,93 & $6,80 \mathrm{E}-04$ & 0,3356 & 1,20 \\
\hline 3-diclorobenzeno & 37,49 & 0,83 & 59,92 & $6,61 \mathrm{E}-04$ & 0,3188 & 1,44 \\
\hline 1,4-diclorobenzeno & 40,29 & 0,94 & 57,32 & $6,30 \mathrm{E}-04$ & 0,3099 & 1,14 \\
\hline 2,4-dinitrofenol & 0,33 & 27,98 & 70,26 & $3,65 \mathrm{E}-04$ & 1,2771 & 0,15 \\
\hline 2-clorofenol & 3,71 & 16,30 & 78,86 & $6,40 \mathrm{E}-04$ & 0,9284 & 0,20 \\
\hline 3,3'-diclorobenzidina & 0,01 & 1,38 & 95,89 & $1,06 \mathrm{E}-03$ & 0,5124 & 2,21 \\
\hline Antraceno & 52,94 & 0,14 & 39,26 & 4,39E-04 & 0,1957 & 7,46 \\
\hline Benzo(a)antraceno & 0,41 & 0,05 & 20,49 & $2,30 \mathrm{E}-04$ & 0,1012 & 78,95 \\
\hline Benzidina & 0,09 & 33,98 & 64,35 & $2,08 \mathrm{E}-04$ & 1,4489 & 0,14 \\
\hline Benzo(a)pireno & 1,11 & 0,02 & 9,85 & $1,10 \mathrm{E}-04$ & 0,0487 & 88,97 \\
\hline Benzo(b)fluoranteno & 0,08 & 0,05 & 19,82 & $2,22 \mathrm{E}-04$ & 0,0979 & 79,96 \\
\hline Benzo(k)fluoranteno & 8,18 & 0,02 & 9,53 & $1,07 \mathrm{E}-04$ & 0,0471 & 82,22 \\
\hline Dibenzo(a,h)antraceno & 1,02 & 0,01 & 2,56 & $2,87 \mathrm{E}-05$ & 0,0126 & 96,40 \\
\hline Difenilnitrosamina & 0,07 & 2,93 & 95,44 & $1,03 \mathrm{E}-03$ & 0,5621 & 1,00 \\
\hline Hexaclorobenzeno & 6,57 & 0,05 & 20,30 & $2,28 \mathrm{E}-04$ & 0,1003 & 72,99 \\
\hline Hexaclorobutadieno & 9,09 & 0,19 & 64,36 & $7,21 \mathrm{E}-04$ & 0,3192 & 26,05 \\
\hline Hexaclorociclopentadieno & 10,71 & 0,13 & 51,23 & $5,74 \mathrm{E}-04$ & 0,2535 & 37,67 \\
\hline Hexacloroetano & 17,38 & 0,38 & 74,84 & $8,36 \mathrm{E}-04$ & 0,3766 & 7,02 \\
\hline Hidrazobenzeno & 0,00 & 4,29 & 94,42 & $9,97 \mathrm{E}-04$ & 0,6025 & 0,69 \\
\hline Indeno(1,2,3-cd)pireno & 2,85 & 0,01 & 2,81 & $3,15 \mathrm{E}-05$ & 0,0139 & 94,32 \\
\hline Isoforona & 3,60 & 26,44 & 68,59 & $3,70 \mathrm{E}-04$ & 1,2176 & 0,15 \\
\hline Naftaleno & 14,76 & 1,78 & 81,80 & $8,93 \mathrm{E}-04$ & 0,4571 & 1,21 \\
\hline Nitrobenzeno & 10,60 & 21,48 & 66,73 & $4,24 \mathrm{E}-04$ & 1,0427 & 0,15 \\
\hline n-nitrosodipropilamina & 3,66 & 32,47 & 62,34 & $2,08 \mathrm{E}-04$ & 1,3889 & 0,14 \\
\hline Pentaclorofenol & 0,00 & 0,13 & 52,87 & $5,93 \mathrm{E}-04$ & 0,2616 & 46,73 \\
\hline Fenantreno & 2,04 & 0,30 & 81,42 & $9,11 \mathrm{E}-04$ & 0,4059 & 15,83 \\
\hline Fenol & 0,22 & 32,03 & 66,21 & $2,58 \mathrm{E}-04$ & 1,3931 & 0,14 \\
\hline Pireno & 4,03 & 0,18 & 63,27 & 7,09E-04 & 0,3135 & 32,21 \\
\hline
\end{tabular}


Tabela 3. Coeficiente de bioconcentração em organismos aquáticos (BCF), raízes (RCF) e seiva de xilema (TSCF) de plantas, coeficiente de sorção no solo $\left(\mathrm{K}_{\mathrm{oc}}\right)$, coeficientes de partição folha-ar $\left(\mathrm{K}_{\mathrm{la}}\right)$ e ar-água $\left(\mathrm{K}_{\mathrm{aw}}\right)$ e o índice GUS dos vinte e nove poluentes orgânicos encontrados em amostras dos lodos de esgotos de Barueri e de Suzano, SP.

\begin{tabular}{|c|c|c|c|c|c|c|c|}
\hline Poluente & $\mathrm{BCF}$ & $\mathrm{RCF}$ & TSCF & $\mathrm{K}_{\mathrm{oc}}\left(\mathrm{m}^{3} \mathrm{~kg}^{-1}\right)$ & $\mathrm{K}_{\mathrm{la}}$ & $\mathrm{K}_{\mathrm{aw}}$ & GUS \\
\hline 1,2,4-triclorobenzeno ${ }^{(1)}$ & 355 & 40 & 0,0967 & $4,51 \mathrm{E}+00$ & $1,95 \mathrm{E}+03$ & $9,17 \mathrm{E}-02$ & 0,50 \\
\hline 1,2-diclorobenzeno ${ }^{(1)}$ & 122 & 14 & 0,2569 & $1,11 \mathrm{E}+00$ & $5,01 \mathrm{E}+02$ & $6,90 \mathrm{E}-02$ & 2,16 \\
\hline 3-diclorobenzeno ${ }^{(1)}$ & 145 & 17 & 0,2235 & $1,39 \mathrm{E}+00$ & $4,44 \mathrm{E}+02$ & $1,36 \mathrm{E}-01$ & 1,93 \\
\hline 1,4-diclorobenzeno ${ }^{(1)}$ & 124 & 14 & 0,2534 & $1,13 \mathrm{E}+00$ & $4,07 \mathrm{E}+02$ & $1,69 \mathrm{E}-01$ & 2,42 \\
\hline 2,4-dinitrofenol $(2,3,4)$ & 6 & 1 & 0,7801 & $1,92 \mathrm{E}-02$ & $4,08 \mathrm{E}+05$ & $1,38 \mathrm{E}-06$ & 6,57 \\
\hline 2-clorofenol ${ }^{(1,2)}$ & 13 & 2 & 0,7412 & $5,81 \mathrm{E}-02$ & $6,51 \mathrm{E}+03$ & $1,55 \mathrm{E}-03$ & 1,07 \\
\hline 3,3'-diclorobenzidina ${ }^{(4,5,6,7)}$ & 140 & 16 & 0,2299 & $1,33 \mathrm{E}+00$ & $3,96 \mathrm{E}+10$ & $1,12 \mathrm{E}-06$ & 1,98 \\
\hline Antraceno $^{(1,4,5,6,8)}$ & 728 & 81 & 0,0422 & $1,16 \mathrm{E}+01$ & $7,98 \mathrm{E}+04$ & $5,90 \mathrm{E}-04$ & $-0,17$ \\
\hline Benzo(a)antraceno ${ }^{(1,4,5,6,8)}$ & 7.204 & 823 & 0,0012 & $2,37 \mathrm{E}+02$ & $5,85 \mathrm{E}+05$ & $2,48 \mathrm{E}-03$ & $-3,80$ \\
\hline Benzidina $^{(2,4,7)}$ & 3 & 1 & 0,7242 & $8,99 \mathrm{E}-03$ & $4,85 \mathrm{E}+08$ & $2,76 \mathrm{E}-08$ & 2,75 \\
\hline Benzo(a)pireno ${ }^{(4,5,6,7,8)}$ & 13.766 & 1.586 & 0,0003 & $5,54 \mathrm{E}+02$ & $1,56 \mathrm{E}+07$ & $4,60 \mathrm{E}-05$ & $-4,75$ \\
\hline Benzo(b)fluoranteno ${ }^{(4,5,6,7,8)}$ & 7.461 & 853 & 0,0011 & $2,48 \mathrm{E}+02$ & $1,07 \mathrm{E}+07$ & $4,53 \mathrm{E}-03$ & $-3,93$ \\
\hline Benzo(k)fluoranteno ${ }^{(4,5,6,7,8)}$ & 13.292 & 1.531 & 0,0004 & $5,29 \mathrm{E}+02$ & $1,22 \mathrm{E}+07$ & $1,64 \mathrm{E}-05$ & $-5,73$ \\
\hline Dibenzo(a,h)antraceno ${ }^{(4,5,6,7,8)}$ & 40.738 & 4.760 & 0,0000 & $2,31 \mathrm{E}+03$ & $5,86 \mathrm{E}+07$ & $6,02 \mathrm{E}-07$ & $-7,09$ \\
\hline Difenilnitrosamina $^{(1)}$ & 72 & 9 & 0,3715 & $5,54 \mathrm{E}-01$ & $4,33 E+05$ & $3,05 \mathrm{E}-02$ & 2,30 \\
\hline Hexaclorobenzeno ${ }^{(1,4,5,6,8)}$ & 6.836 & 781 & 0,0013 & $2,21 \mathrm{E}+02$ & $4,12 \mathrm{E}+03$ & $4,45 \mathrm{E}-02$ & $-4,54$ \\
\hline Hexaclorobutadieno $^{(1,5,6,8)}$ & 1.297 & 146 & 0,0196 & $2,48 \mathrm{E}+01$ & $5,42 \mathrm{E}+02$ & $9,65 \mathrm{E}-01$ & $-0,89$ \\
\hline Hexaclorociclopentadieno ${ }^{(1,5,6,8)}$ & 2.044 & 230 & 0,0101 & $4,51 \mathrm{E}+01$ & $2,31 \mathrm{E}+02$ & $4,89 \mathrm{E}-01$ & $-0,95$ \\
\hline Hexacloroetano $^{(1)}$ & 423 & 47 & 0,0800 & $5,67 \mathrm{E}+00$ & $8,14 \mathrm{E}+02$ & $5,35 \mathrm{E}-02$ & 0,57 \\
\hline Hidrazobenzeno $^{(2,3,4,7)}$ & 52 & 6 & 0,4517 & $3,58 \mathrm{E}-01$ & $8,02 \mathrm{E}+07$ & $1,96 \mathrm{E}-05$ & 3,26 \\
\hline Indeno(1,2,3-cd)pireno ${ }^{(4,5,6,7,8)}$ & 37.325 & 4.356 & 0,0000 & $2,06 \mathrm{E}+03$ & $2,07 \mathrm{E}+07$ & $9,78 \mathrm{E}-06$ & $-6,70$ \\
\hline Isoforona $^{(2,3)}$ & 6 & 1 & 0,7819 & $2,06 \mathrm{E}-02$ & $5,49 \mathrm{E}+03$ & $2,71 \mathrm{E}-04$ & 3,89 \\
\hline Naftaleno $^{(1)}$ & 97 & 11 & 0,3042 & $8,20 \mathrm{E}-01$ & $1,69 \mathrm{E}+03$ & $1,89 \mathrm{E}-02$ & 1,83 \\
\hline Nitrobenzeno ${ }^{(1,2,3)}$ & 8 & 2 & 0,7824 & $2,91 \mathrm{E}-02$ & $1,86 \mathrm{E}+03$ & $7,77 \mathrm{E}-04$ & 5,82 \\
\hline n-nitrosodipropilamina $a^{(2,3,4)}$ & 3 & 1 & 0,7293 & $9,42 \mathrm{E}-03$ & $4,74 \mathrm{E}+03$ & $4,63 \mathrm{E}-05$ & 6,82 \\
\hline Pentaclorofenol ${ }^{(4,7,8)}$ & 2.351 & 265 & 0,0081 & $5,42 \mathrm{E}+01$ & $2,60 \mathrm{E}+08$ & $1,13 \mathrm{E}-04$ & $-1,65$ \\
\hline Fenantreno $^{(1,3,5,6,8)}$ & 741 & 83 & 0,0413 & $1,19 \mathrm{E}+01$ & $1,06 \mathrm{E}+05$ & $9,34 \mathrm{E}-04$ & $-0,17$ \\
\hline Fenol $^{(2,3,4)}$ & 4 & 1 & 0,7518 & $1,19 \mathrm{E}-02$ & $8,37 \mathrm{E}+04$ & $2,14 \mathrm{E}-05$ & 2,93 \\
\hline Pireno $^{(4,5,6,8)}$ & 1.545 & 174 & 0,0153 & $3,12 \mathrm{E}+01$ & $4,92 \mathrm{E}+05$ & $3,63 \mathrm{E}-04$ & $-1,64$ \\
\hline
\end{tabular}

${ }^{(1)}$ Potencial poluente do ar. ${ }^{(2)}$ Potencial poluente da seiva do xilema. ${ }^{(3)}$ Potencial poluente da água subterrânea. ${ }^{(4)}$ Potencial poluente de folhas pelo ar. (5)Potencial poluente da biota aquática. ${ }^{6}$ Potencial poluente de raízes de plantas. (7)Potencial poluente de folhas por deposição de partículas contaminadas. ${ }^{(8)}$ Potencial poluente da matriz sólida do solo.

\section{Conclusões}

1. A modelagem da distribuição ambiental de vinte e nove poluentes orgânicos de lodos de esgoto indica quais os compartimentos ambientais preferenciais dos poluentes.

2. Os fatores de bioconcentração BCF, RCF e TSCF, os coeficientes de partição ar-água e folha-ar e o índice GUS indicam o grau de afinidade entre os poluentes orgânicos dos lodos de esgoto e os compartimentos ar, água, solo, planta e biota aquática.

\section{Referências}

ANDRADE, C.A.; MATTIAZZO, M.E. Nitratos e metais pesados no solo e nas árvores após aplicação de biossólidos (lodo de esgotos) em plantações florestais de Eucalyptus grandis. Scientia Forestalis, v.58, p.59-72, 2000.

BACCI, E. Ecotoxicology of organic contaminants. Boca Raton: Lewis Publishers, 1994. 165p.
BOEIRA, R.C.; LIGO, M.A.V.; DYNIA, J.F. Nitrogen mineralization in a tropical soil amended with sewage sludges. Pesquisa Agropecuária Brasileira, v.37, p.1639-1647, 2002.

BRIGGS, G.G.; BROMILOW, R.H.; EVANS, A.A. Relationships between lipophilicity and root uptake and translocation of nonionised chemicals by barley. Pesticide Science, v.13, p.495-504, 1982.

BRIGGS, G.G.; BROMILOW, R.H.; EVANS, A.A. Relationships between lipophilicity and the distribution of non-ionized chemicals in barley shoots following uptake by the roots. Pesticide Science, v.14, p.492-500, 1983.

COUSINS, I.T.; MACKAY, D. Strategies for including vegetation compartments in multimedia models. Chemosphere, v.44, p.643654, 2001.

GUSTAFSON, D.I. Groundwater ubiquity score: a simple method for assessing pesticide leachability. Environmental Toxicology and Chemistry, v.8, p.339-357, 1989.

HOWARD, P.H.; BOETHLING, R.S.; JARVIS, W.F.; MEYLAN, W.M.; MICHALENKO, E.M. Handbook of environmental degradation rates. Chelsea: Lewis Publishers, 1991. 725p. 
ISNARD, P.; LAMBERT, S. Estimating bioconcentration factors from octanol-water partition-coefficient and aqueous solubility. Chemosphere, v.17, p.21-34, 1988.

KARICKHOFF, S.W. Semi-empirical estimation of sorption of hydrophobic pollutants on natural sediments and soils. Chemosphere, v.10, p.833-846, 1981.

MACKAY, D. Multimedia environmental models: the fugacity approach. Chelsea: Lewis Publishers, 1991. 257p.

MACKAY, D.; PATERSON, S.; DI GUARDO, A.; COWAN, C.E. Evaluating the environmental fate of a variety of types of chemicals using the EQC model. Environmental Toxicology and Chemistry, v.15, p.1627-1637, 1996.

OECD. Organization for Economic Cooperation and Development. Environmental exposure assessment strategies for existing industrial chemicals in OECD member countries. Paris: OECD, 1999. 31p. (OECD Series on Testing and Assessment, 17).

OECD. Organization for Economic Cooperation and Development. Guidelines for testing of chemicals: bioaccumulation sequential statistic fish test. Paris: OECD, 1981.

PARAIBA, L.C.; CARRASCO, J.M.; BRU, R. Level IV fugacity model by a continuos time control system. Chemosphere, v.38, p.1763-1775, 1999.

PEREIRA NETTO, A.D.; MOREIRA, J.C.; DIAS, A.E.X.; ARBILLA, G.; FERREIRA, L.F.V.; OLIVEIRA, A.S.; BAREK, J. Avaliação da contaminação humana por hidrocarbonetos policíclicos aromáticos (HPAS) e seus derivados nitrados (NHPAS): uma revisão metodológica. Química Nova, v.23, p.265-273, 2000.
RAND, G.M.; WELLS, P.G.; MCCARTY, L.S. Introduction to aquatic toxicology. In: RANDY, M.G. (Ed.). Fundamentals of aquatic toxicology: effects, environmental fate, and risk assessment. Florida: Taylor \& Francis, 1995. p.3-67.

ROCHA, R.E.M.; PIMENTEL, M.S.; ZAGO, V.C.P.; RUMJANEK, N.G.; DE-POLLI, H. Avaliação de biossólido de águas servidas domiciliares como adubo em couve. Pesquisa Agropecuária Brasileira, v.38, p.1435-1441, 2003.

SRC. Syracuse Research Corporation [on line]. Disponível em: <http://www.syrres.com/esc/physdemo.htm.> Acesso em: $21 \mathrm{fev}$. 2005.

TRAPP, S.; MCFARLANE, J.C. Plant contamination: modeling and simulation of organic chemical processes. Boca Raton: CRC Press, 1995. 254p.

TRAPP, S.; MATTHIES, M. Chemodynamics and environmental modeling. Heidelberg: Springer, 1998. 285p.

TRAPP, S.; MATTHIES, M. Generic one-compartment model for the uptake of organic chemicals by foliar vegetation. Environmental Science and Technology, v.29, p.2333-2338, 1995.

TSUTIYA, M.T. Características de biossólidos gerados em estações de tratamento de esgotos. In: TSUTIYA, M.T.; COMPARINI, J.B.; SOBRINHO, A.P.; HESPANOL, I.; CARVALHO, P.C.T.; MELFI, A.J. (Ed.). Biossólidos na agricultura. São Paulo: Sabesp, 2001. p.89-131.

VIEIRA, R.F.; CARDOSO, A.A. Variações nos teores de nitrogênio mineral em solo suplementado com lodo de esgoto. Pesquisa Agropecuária Brasileira, v.38, p.867-874, 2003.

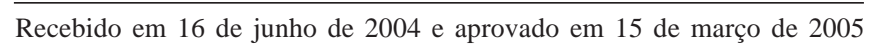

